\title{
Phase lag deduced information in photo-thermal actuation for nano-mechanical systems characterization
}

\author{
R. J. F. Bijster, ${ }^{1, a)}$ J. de Vreugd, ${ }^{1}$ and H. Sadeghian ${ }^{1,2}$ \\ ${ }^{1}$ Department of Optomechatronics, TNO, Stieltjesweg 1,2628 CK Delft, The Netherlands \\ ${ }^{2}$ Department of Precision and Microsystems Engineering, Delft University of Technology, 2628 CD Delft, \\ The Netherlands
}

(Received 3 July 2014; accepted 7 August 2014; published online 18 August 2014)

In photo-thermal actuation, heat is added locally to a micro-cantilever by means of a laser. A fraction of the irradiation is absorbed, yielding thermal stresses and deformations in the structure. Harmonic modulation of the laser power causes the cantilever to oscillate. Moreover, a phase lag is introduced which is very sensitive to the spot location and the cantilever properties. This phase lag is theoretically predicted and experimentally verified. Combined with thermo-mechanical properties of the cantilever and its geometry, the location of the laser spot, the thermal diffusivity, and the layer thicknesses of the cantilever can be extracted. (C) 2014 AIP Publishing LLC.

[http://dx.doi.org/10.1063/1.4893461]

Nano-cantilevers are used in applications from scanning probe microscopy ${ }^{1}$ to chemical analysis ${ }^{2,3}$ and bio-sensing. ${ }^{4}$ The principle is based on measuring changes in the motion of a micro-cantilever that are caused by the interaction with the subject. This is typically done using the optical beam deflection (OBD) method. ${ }^{5}$ In this method, a laser is focused on the cantilever and the reflected beam is registered on a position sensitive detector. A fraction of the irradiation, however, is absorbed and causes a temperature gradient over the length of the cantilever that results in thermal stresses and deformations. Through modulation of the absorbed power, the cantilever can be actuated; a technique which is known as photo-thermal actuation. ${ }^{6-9}$ Because the heat is absorbed in only a small area, time is required to dissipate the heat through the structure and for the temperature gradient to change. The dissipation time results in a phase lag between the actuation signal and the thermo-mechanical response which can be predicted theoretically based on material properties and the geometry. The phase lag information can be used for extraction of both thermal and mechanical properties of the cantilever and the location of the laser spot along its length. In this Letter, the methodology and its applications are outlined.

Photo-thermal actuation relies on temperature gradient induced stresses that cause deformation of a microcantilever. The temperature gradient along the length of a cantilever is described by the one-dimensional heat equation. Given in its general form

$$
\frac{\partial T(x, t)}{\partial t}=D \frac{\partial^{2} T(x, t)}{\partial x^{2}}-B\left(T(x, t)-T_{e n v}\right)+f(x, t),
$$

it describes the temperature $T$ as a function of time $t$, location along the cantilever $x$, the thermal diffusivity $D\left(\mathrm{~m}^{2} \mathrm{~s}^{-1}\right)$, the characteristic time constant for convective heat transfer $B$ $\left(\mathrm{s}^{-1}\right)$, the ambient temperature $T_{e n v}$, and forcing function $f$.

\footnotetext{
a)Electronic mail: roy.bijster@tno.nl
}

The base of the cantilever and its tip are defined to be at $x=0$ and at $x=L$, respectively.

From a thermomechanical perspective, a cantilever beam is thermally isolated at the tip and has a heat sink at its base. Mathematically these boundary conditions are represented by a Dirichlet and a Neumann boundary condition at the base and tip, respectively. The forcing function describes the absorbed power along the length of the cantilever and is represented by a Gaussian distribution as follows:

$$
f(x, t)=a(t) \exp \left(-\frac{(x-b)^{2}}{2 c^{2}}\right),
$$

where $a$ is the absorbed laser power, $b$ is the distance from the base to the center of the spot, and $c$ is the width of the spot. Using these conditions, a closed form solution to Eq. (1) can be found.

The temperature distribution is coupled to the mechanical response. Cantilevers that are typically used in OBD setups have a thick silicon based substrate. Because this substrate has a low reflectivity, a (gold) reflective layer is added to increase the signal-to-noise ratio of the OBD method. The thermomechanical response of the cantilever is a function of the mismatch strain that occurs at the interface between the layers as a result from the temperature distribution. ${ }^{10}$ It is assumed that thermal gradients are limited to the longitudinal direction. The cantilever is assumed to be in quasi-steady state. Therefore, the influence of inertial effects and mechanical damping are neglected. Only the period and phase of the mechanical oscillation are considered. As a result of these simplifications, only the thermal properties of the cantilever materials are required. The transfer function that couples the heat input to the mechanical response is solved in the Laplace domain and uses the zero rotation boundary condition at the base $(\Theta(x=0, s)=0)$. The full derivation of the transfer function is given elsewhere, ${ }^{11}$ but the final result is repeated here. It is the fraction of the forcing function in the Laplace domain $F(s)$ and the resulting pseudo-rotation $\Theta(s)$ 


$$
\begin{aligned}
G(s)=\left.\frac{F(s)}{\Theta(s)}\right|_{x=b}= & \frac{\sqrt{2 \pi} c}{8(s+B) \cosh \left(k_{3}\right)}\left\{-2 \exp \left(k_{1}\right)\left[\operatorname{erf}\left(\mathrm{m}_{1}\right)+\operatorname{erf}\left(\mathrm{m}_{2}\right)\right]+2 \exp \left(k_{2}\right)\left[\operatorname{erf}\left(\mathrm{m}_{4}\right)-\operatorname{erf}\left(\mathrm{m}_{3}\right)\right]\right. \\
& +4 \cosh \left(k_{3}\right) \operatorname{erf}\left(\mathrm{m}_{5}\right)+\exp \left(k_{4}\right)\left[\operatorname{erf}\left(\mathrm{m}_{3}\right)-\operatorname{erf}\left(\mathrm{m}_{4}\right)\right]+\exp \left(k_{5}\right)\left[\operatorname{erf}\left(\mathrm{m}_{2}\right)+\operatorname{erf}\left(\mathrm{m}_{4}\right)\right] \\
& \left.+\exp \left(k_{6}\right)\left[\operatorname{erf}\left(\mathrm{m}_{3}\right)-\operatorname{erf}\left(\mathrm{m}_{1}\right)\right]+\exp \left(k_{7}\right)\left[\operatorname{erf}\left(\mathrm{m}_{1}\right)+\operatorname{erf}\left(\mathrm{m}_{2}\right)\right]\right\}
\end{aligned}
$$

where the auxiliary parameters $m_{i}$ and $k_{i}$ describe the thermal properties of the cantilever and of the laser spot $(D, b, L$, and $c)$. These parameters are available in the supplementary material. ${ }^{12}$ From the transfer function can be concluded that the phase lag is dependent on the laser spot location and the driving frequency of the laser. Because the location of the laser spot coincides with the location where the response is measured, it follows that $x=b$. Using $s=i \omega$ in the transfer function, the phase lag angle can be derived for a given spot location and a harmonic input of frequency $\omega$. The generic results are presented in Fig. 1. From the figure, it is concluded that a unique set of phase shifts can be mapped to each set of frequencies depending on the laser spot location.

This unique phase lag profile allows localization of the laser spot along the cantilever length. This can be used for automatic alignment of a probe in, e.g., a SPM setup. Accurate alignment using photo-thermal actuation allows for maximization of the sensitivity of a measurement in an existing OBD setup, without the need for additional hardware. This concept was verified using a table-top OBD setup ${ }^{13}$ of which a schematic illustration is depicted in Fig. 2. The setup uses a modulated fiber laser of which the beam is focused onto the cantilever by means of the microscope objective. The reflected light passes back through the microscope objective and is imaged on an optical position sensor (OPS). The paths of the illuminating beam and returning beam are separated through polarizing beam splitters. While part of the light is reflected to the OPS, another part is guided further and imaged on a charge-coupled device (CCD) for optical microscopy and manual alignment. The verification encompasses placing the laser spot at several known locations along the length of the cantilever. The spots are centered laterally and their locations are indicated in Fig. 3(a). The actual location was found through correlation of the CCD images with earlier obtained scanning electron

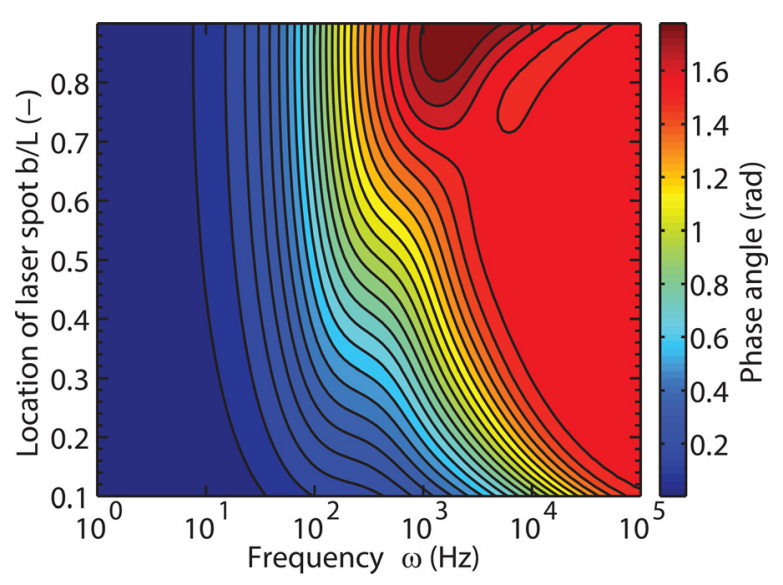

FIG. 1. Phase shift as a function of position and signal frequency. $L=500$ $\mu \mathrm{m}, D=7 \times 10^{-5} \mathrm{~m}^{2} \mathrm{~s}^{-1}$, full-width half minimum of spot $=30 \mu \mathrm{m}$. micrographs. The power of the laser was varied harmonically at frequencies between $1 \mathrm{~Hz}$ and $10 \mathrm{kHz}$. The upper limit was chosen to include the first mechanical resonance frequency of $\approx 6 \mathrm{kHz}$. The measured phase shifts are depicted in Fig. 3(b). The model does not include inertial effects and was therefore not verified in the frequency range above the first mechanical resonance with statistically significant measurements. The spot location was extracted by comparison of the phase lags with the theoretical model for frequencies up to the first resonance frequency. The comparison between the actual locations and the extracted locations of the spot (Fig. 3(c)) supports the validity of the theory. A residual offset is present in the form of an offset in the fit. It is hypothesized that the cause for this difference traces back to the unknown convective heat transfer coefficient and the difference in tip geometry. While the real cantilever has a triangular tip, the model assumes a rectangular plan form.

Another application of the phase lag deduced information is thickness estimation of the layers of the cantilever. These layer thicknesses are hard if not impossible to measure accurately with conventional techniques such as scanning electron microscopy. When the location of the laser spot is known, the phase lag deduced information can be used to estimate the thermal diffusivity. Combined with the effective conductance of the cantilever, the layer thicknesses for a bilayer cantilever can be determined. The thermal diffusivity is approximated as a function of the layer conductance $k_{i}$, thickness $t_{i}$, heat capacity $c_{p_{i}}$, and density $\rho_{i}$, as follows:

$$
D=\frac{k_{1} t_{1}+k_{2} t_{2}}{c_{p_{1}} \rho_{1} t_{1}+c_{p_{2}} \rho_{2} t_{2}} .
$$

Similarly, the effective conductance $G$ is expressed as a function of the layer conductance and thickness, the cantilever width $w$, and length $L$ as follows:

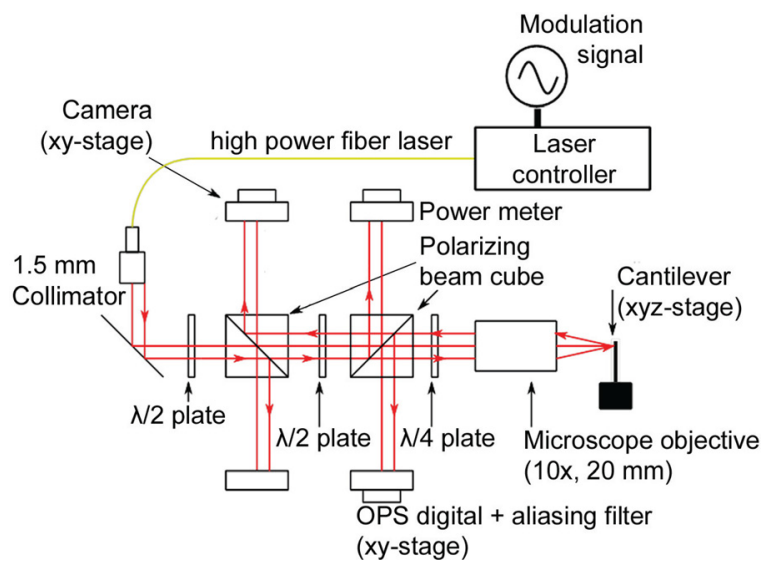

FIG. 2. Schematic illustration of the table-top OBD setup. Reproduced by permission from Herfst et al., Measurement 56, 104-106 (2014). Copyright 2014 by Elsevier. 


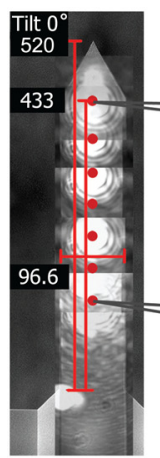

(a)

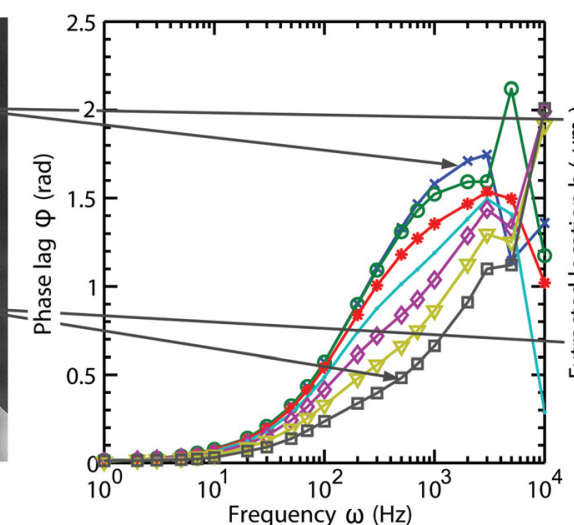

(b)

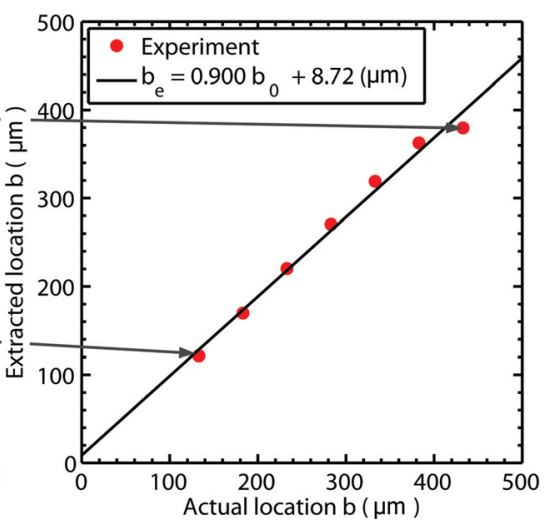

(c)

$$
G=\left(k_{1} t_{1}+k_{2} t_{2}\right) \frac{w}{L} .
$$

Combined, Eqs. (4) and (5) result in a set of two equations and two unknowns, which can be solved for the layer thicknesses. This method relies on an accurate estimate of the thermal diffusivity and the effective conductance. The former can be determined from the phase lag information, if the time constant for convective heat transfer $B$ is known. Its value is related to the surface area $A$ that is involved in heat exchange with the environment, the heat transfer coefficient $h$ and the heat capacity $C$. For a thin cantilever with $t \ll L$, $w$, this is approximated as

$$
B=\frac{h A}{C} \approx \frac{2 h}{c_{p_{1}} \rho_{1} t_{1}+c_{p_{2}} \rho_{2} t_{2}} .
$$

For cantilevers with dimensions in the micrometer scale, a wide range of values for the effective convective heat transfer coefficient $h$ is reported. Based on modeling and experimental evidence typical values of $30 \mathrm{~W} \mathrm{~m}^{-2} \mathrm{~K}^{-1}$ to $10 \mathrm{~kW}$ $\mathrm{m}^{-2} \mathrm{~K}^{-1}$ have been predicted and reported. ${ }^{14-19}$ The physical mechanism of heat exchange with the surrounding air on the microscale is not sufficiently well understood to explain this range and is expected to be a combination of gas conduction and natural convection and to be dependent on the geometry as well as the frequency at which the heat is applied. ${ }^{17-19}$

It is experimentally verified that the thermal diffusivity can be extracted from the phase lag information. In the measurement campaign, the NanoWorld ARROW TL8-Au cantilevers were used. These cantilevers have a triangular tip and are composed of a $1 \mu \mathrm{m}$ thick substrate of monolithic silicon and a $30 \mathrm{~nm}$ thick gold coating. An intermediate $5 \mathrm{~nm}$ layer of titanium is present to limit diffusion of the gold molecules into the substrate. By combining the manufacturer specifications with published values for the substrate and coating materials, ${ }^{20-25}$ the thermal diffusivity is estimated to be $7 \times 10^{-5}$ $\mathrm{m}^{2} \mathrm{~s}^{-1}$. Using the phase lag information, it was found that an effective value of $h \approx 340 \mathrm{~W} \mathrm{~m}^{-2} \mathrm{~K}^{-1}$ yields an extracted value for the thermal diffusivity that is in agreement with the theoretical estimate. The used value of the effective heat transfer coefficient $h$ falls well within the range of reported values. The actual value could not be determined with the experimental setup. Future experiments at vacuum conditions will have to provide additional information on the implementation of this technique by eliminating this issue. In addition to the thermal diffusivity, also the effective conductance needs to be measured. This can be done by studying the effect of an increased heat load and a varying base temperature on the deformation of the cantilever. ${ }^{11,18}$

In conclusion, two applications are proposed that utilize information that is extracted from the phase lag information that is obtained through the photo-thermal actuation method for nano-mechanical cantilevers. The information is used to estimate the laser spot location along the length of the cantilever and to estimate thermal properties. From the thermal properties and known material properties, geometric details such as the layer thicknesses of bi-layer cantilevers can be estimated. Future research will focus on repeating the validation experiments under vacuum conditions and in finding an adequate model to represent the heat exchange with the environment.

This research was financially supported by enabling technology program Optomechatronics, TNO.

${ }^{1}$ N. Umeda, S. Ishizaki, and H. Uwai, "Scanning attractive force microscope using photothermal vibration," J. Vac. Sci. Technol., B 9, 1318 (1991).

${ }^{2}$ T. Thundat, R. J. Warmack, G. Y. Chen, and D. P. Allison, "Thermal and ambient-induced deflections of scanning force microscope cantilevers," Appl. Phys. Lett. 64, 2894 (1994).

${ }^{3}$ M. Varshney, P. S. Waggoner, C. P. Tan, K. Aubin, R. A. Montagna, and H. G. Craighead, "Prion protein detection using nanomechanical resonator arrays and secondary mass labeling," Anal. Chem. 80, 2141-2148 (2008).

${ }^{4}$ A. Gupta, D. Akin, and R. Bashir, "Single virus particle mass detection using microresonators with nanoscale thickness," Appl. Phys. Lett. 84, 1976 (2004).

${ }^{5}$ G. Meyer and N. M. Amer, "Novel optical approach to atomic force microscopy,” Appl. Phys. Lett. 53, 1045 (1988).

${ }^{6} \mathrm{~S}$. Venkatesh and B. Culshaw, "Optically activated vibrations in a micromachined silica structure," Electron. Lett. 21, 315 (1985).

${ }^{7}$ T. Lammerink, M. Elwenspoek, and J. Fluitman, "Optical excitation of micro-mechanical resonators," in Proceedings of IEEE Micro Electro Mechanical Systems (IEEE, Nara, 1991), pp. 160-165.

${ }^{8} \mathrm{~S}$. Inaba, K. Akaishi, T. Mori, and K. Hane, "Analysis of the resonance characteristics of a cantilever vibrated photothermally in a liquid," J. Appl. Phys. 73, 2654 (1993).

${ }^{9}$ A. Labuda, K. Kobayashi, Y. Miyahara, and P. Grütter, "Retrofitting an atomic force microscope with photothermal excitation for a clean cantilever response in low Q environments." Rev. Sci. Instrum. 83, 053703 (2012).

${ }^{10}$ W. Young, R. G. Budynas, and A. Sadegh, Roark's Formulas for Stress and Strain, 8th ed. (McGraw-Hill, New York, 2011).

${ }^{11}$ R. Bijster, J. de Vreugd, and H. Sadeghian, "Dynamic characterization of Bimaterial cantilevers," in SENSORDEVICES 2013: The Fourth International 
Conference on Sensor Device Technologies and Applications, edited by S. Yurish and F. Pacull (IARIA, Barcelona, Spain, 2013), pp. 1-8.

${ }^{12}$ See supplementary material at http://dx.doi.org/10.1063/1.4893461 for the transfer function with the auxiliary parameters.

${ }^{13}$ R. Herfst, W. Klop, M. Eschen, T. van den Dool, N. Koster, and H. Sadeghian, "Systematic characterization of optical beam deflection measurement system for micro and nanomechanical systems," Measurement 56, 104-106 (2014).

${ }^{14}$ J. Peirs, D. Reynaerts, and H. Van Brussel, "Scale effects and thermal considerations for micro-actuators," in Proceedings of 1998 IEEE International Conference on Robotics and Automation (IEEE, Leuven, Belgium, 1998), Vol. 2, pp. 1516-1521.

${ }^{15}$ K. Park, J. Lee, Z. M. Zhang, and W. P. King, "Frequency-dependent electrical and thermal response of heated atomic force microscope cantilevers," J. Microelectromech. Syst. 16, 213-222 (2007).

${ }^{16}$ X. J. Hu, A. Jain, and K. E. Goodson, "Investigation of the natural convection boundary condition in microfabricated structures," Int. J. Therm. Sci. 47, 820-824 (2008)

${ }^{17}$ J. Lee, F. Goericke, and W. P. King, "Temperature-dependent thermomechanical noise spectra of doped silicon microcantilevers," Sens. Actuators, A 145-146, 37-43 (2008)
${ }^{18}$ S. Shen, A. Narayanaswamy, S. Goh, and G. Chen, "Thermal conductance of bimaterial microcantilevers," Appl. Phys. Lett. 92, 063509 (2008).

${ }^{19}$ K. J. Kim and W. P. King, "Thermal conduction between a heated microcantilever and a surrounding air environment," Appl. Therm. Eng. 29, 1631-1641 (2009).

${ }^{20}$ B. Bardes, H. Baker, W. Cubberly, and ASM International Committee, Metals Handbook, 9th ed., edited by B. Bardes, P. Baker, W. H. Cubberly et al. (American Society for Metals, 1978).

${ }^{21}$ J.-H. Jou, C.-N. Liao, and K.-W. Jou, "A method for the determination of gold thin film's mechanical properties," Thin Solid Films 238, 70-72 (1994).

${ }^{22}$ Y. S. Ju, "Phonon heat transport in silicon nanostructures," Appl. Phys. Lett. 87, 153106 (2005).

${ }^{23} \mathrm{~W}$. Liu and M. Asheghi, "Phonon-boundary scattering in ultrathin singlecrystal silicon layers," Appl. Phys. Lett. 84, 3819 (2004).

${ }^{24}$ S. Okuda, M. Kobiyama, and T. Inami, "Mechanical properties and thermal stability of nanocrystalline gold prepared by gas deposition method," Mater. Trans. JIM 40, 412-415 (1999).

${ }^{25}$ D. Son, J.-h. Jeong, and D. Kwon, "Film-thickness considerations in microcantilever-beam test in measuring mechanical properties of metal thin film," Thin Solid Films 437, 182-187 (2003). 